\title{
Artefact
}

Techniques, histoire et sciences humaines

Les circulations de savoir et les transferts de techniques d'irrigation entre populations arabe et juive en Palestine ottomane et mandataire (fin XIX ${ }^{\mathrm{e}}$ siècle-1948)

The Circulation of Knowledge and the Transfer of Irrigation Techniques between Arab and Jewish Populations in Ottoman and Mandatory Palestine (end of the 19th century-1948)

\section{Élisabeth Mortier}

\section{(2) OpenEdition}

1 Journals

\section{Édition électronique}

URL : http://journals.openedition.org/artefact/5842

DOI : $10.4000 /$ artefact.5842

ISSN : 2606-9245

\section{Éditeur :}

Association Artefact. Techniques histoire et sciences humaines, Presses universitaires du Midi

\section{Édition imprimée}

Date de publication : 15 juillet 2020

Pagination : 231-253

ISBN : 978-2-8107-0691-4

ISSN : 2273-0753

Référence électronique

Élisabeth Mortier, « Les circulations de savoir et les transferts de techniques d'irrigation entre populations arabe et juive en Palestine ottomane et mandataire (fin xIx siècle-1948) », Artefact [En ligne], 12 | 2020, mis en ligne le 21 décembre 2020, consulté le 23 décembre 2020. URL : http:// journals.openedition.org/artefact/5842 ; DOI : https://doi.org/10.4000/artefact.5842

\section{(c) (†) $\odot$}

Artefact, Techniques, histoire et sciences humaines est mise à disposition selon les termes de la Licence Creative Commons Attribution - Pas d'Utilisation Commerciale - Pas de Modification 4.0 International. 


\section{Les circulations de savoir et les transferts de techniques d'irrigation entre populations arabe et juive en Palestine ottomane et mandataire (fin XIX ${ }^{\mathrm{e}}$ siècle-1948)}

Élisabeth Mortier

\section{Résumé}

La fin de la domination ottomane en Palestine et la période mandataire britannique (1922-1948) constituent un moment de circulations et de transferts techniques agricoles entre la population arabe et la population juive. Ces circulations techniques s'opèrent notamment dans le cadre de l'essor de l'irrigation pendant le Mandat britannique. En effet, les procédés de conservation, de pompage et de distribution de l'eau développés par les paysans arabes tout au long de la domination ottomane sont transformés sous l'impulsion de l'import de techniques européennes et nord-américaines par les sionistes. Les techniques arabes, fruits d'échanges pluriséculaires, n'en sont pas moins maintenues pendant le Mandat.

\section{Mots-clés}

sionisme, pompage, shadoof, qanat, sakia, kibboutz, mochav

99 Élisabeth Mortier, «Les circulations de savoir et les transferts de techniques d'irrigation entre populations arabe et juive en Palestine ottomane et mandataire (fin XIXe siècle-1948) », Artefact, 12, 2020, p. 231-253. 


\section{The Circulation of Knowledge and the Transfer of Irrigation Techniques between Arab and Jewish Populations in Ottoman and Mandatory Palestine (end of the 19th century-1948)}

\section{Abstract}

The end of the Ottoman rule over Palestine and the British Mandate period (1922-1948) constituted period of agricultural circulation and technical transfers between the Arab and Jewish populations. These technical circulations took place in particular in the context of the development of irrigation during the British Mandate. Indeed, the processes of water conservation, pumping and distribution developed by Arab peasants throughout the Ottoman domination were transformed under the impulse of Zionists' import of European and North American techniques. Arab techniques, the fruit of centuries of exchanges, were nevertheless maintained during the Mandate.

\section{Keywords}

Zionism, pumping, shadoof, qanat, sakia, kibbutz, mochav 
$\mathrm{E}$ n 1935, le jeune géographe français Jean Gottmann, alors âgé d'une vingtaine d'années, affirme que : «L'irrigation palestinienne actuelle est une réalisation toute récente; l'étudier aujourd'hui c'est la prendre à une phase transitoire de son développement ${ }^{1} »$. Par cette phrase, il fait de la domination britannique en Palestine un moment décisif pour l'essor des cultures irriguées, éludant ainsi des siècles d'utilisation des techniques d'irrigation antiques et arabes sur ce territoire. Pourtant, avant l'installation des premiers colons juifs à la fin du XIx siècle et l'arrivée des Britanniques en 1917, la culture irriguée est déjà très présente en Palestine ottomane avec la culture des agrumes dans la plaine côtière, en particulier l'orange Shamouti ${ }^{2}$, connue sous le nom d'orange de Jaffa, une variété développée par les Arabes au milieu du siècle. Pendant le Mandat, confié par la Société des Nations (SDN) à la Grande-Bretagne en 1922, l'agriculture irriguée se développe considérablement dans le cadre d'une situation politique et sociale complexe. Les Britanniques se trouvent confrontés à deux obligations : ils doivent respecter la promesse faite aux sionistes de favoriser l'établissement d'un " Foyer national juif " en Palestine - promesse de la déclaration Balfour du 2 novembre 1917, inscrite dans la charte du mandat - tout en s'efforçant d'améliorer les conditions de vie et d'expression politique de la population arabe jusqu'à leur départ en $1948^{3}$. L'irrigation des terres constitue le principal usage agricole de l'eau en Palestine. Elle peut se définir comme le fait d'apporter de l'eau, par divers moyens techniques, dans un espace cultivé pour permettre le bon développement des cultures. Les besoins en irrigation varient en fonction de l'aridité des espaces, des besoins hydriques des différentes espèces de plantes cultivées et de l'intensité du rendement agricole souhaité par les hommes. Les apports en eau, indispensables pour l'irrigation, sont loin d'être homogènes en Palestine. Trois types de ressources hydriques sont utilisés pour l'irrigation : les eaux souterraines, les eaux de pluies et les rivières. Les eaux de pluies, qui tombent d'octobre à avril, sont très inégalement réparties dans l'espace palestinien : les zones montagneuses autour de Jérusalem connaissent un taux de pluviométrie important tandis que le sud du pays, le désert du Néguev, est aride. De la même manière, l'hydrographie palestinienne, principalement constituée du Jourdain, du Kishon, dans la baie de

\footnotetext{
1. Gottmann, 1935, p. 148.

2. Nicault, 2014, p. 80.

3. Situation que Nadine Picaudou nomme une " double-obligation " : Picaudou, 2006.
} 
Haïfa, et de l'Auja (Yarkon en hébreu) qui se jette dans la Méditerranée au nord de Tel-Aviv, a des caractéristiques physiques très différentes. En effet, l'eau du Kishon a un taux de salinité qui limite son usage pour l'agriculture irriguée. Quant aux eaux souterraines, sources principales de l'irrigation dans la plaine côtière de la Palestine, elles sont méconnues au moment de la mise en place du Mandat britannique. Les techniques d'irrigation sont donc adaptées en fonction des caractéristiques physiques des espaces agricoles et des ressources en eau disponibles. Il est possible néanmoins de répartir les techniques d'irrigation en trois catégories : les modes de conservation de l'eau, les méthodes de pompage et les procédés de distribution dans les cultures. Nous mettons de côté dans cet article les techniques liées à la recherche de l'eau, comme les forages hydrauliques pour l'irrigation.

L'irrigation est un sujet incontournable des études sur l'histoire agricole de la Palestine. Depuis les années 1990, deux approches principales permettent l'histoire de l'irrigation en Palestine : l'histoire économique des productions agricoles juives et arabes et l'étude de l'agrumiculture de la plaine côtière. Jacob Metzer, professeur israélien d'histoire économique, publie en 1997 une première recherche sur l'économie duale du Mandat britannique entre population arabe majoritaire et communauté juive. Dans cette étude il considère l'irrigation pour son rendement et sa place dans l'économie palestinienne. Les travaux de Charles S. Kamen ${ }^{4}$ et Amos $\mathrm{Nadan}^{5}$ approfondissent la recherche économique sur les cultures irriguées. Les coûts financiers et les capacités d'investissement en sont le centre. Leurs recherches s'inscrivent dans le cadre d'une analyse plus générale des caractéristiques de l'agriculture arabe comparées aux terres cultivées par les Juifs. Dans ces trois publications majeures pour l'histoire économique de l'agriculture palestinienne, il y a peu de place pour l'histoire des techniques d'irrigation. La recherche sur la culture des oranges dans la plaine côtière s'intéresse davantage aux évolutions techniques et aux transferts technologiques qui ont transformé l'irrigation en Palestine. Abordée par Mark Levine dans son travail sur $\mathrm{Jaffa}^{6}$, la culture des oranges par l'irrigation est précisément étudiée par Nahum Karlinsky dans ses travaux ${ }^{7}$. Quant aux transferts techniques entrepris par les Britanniques, Roza El-Eini les

\footnotetext{
4. Kamen, 1991.

5. Nadan, 2006.

6. Levine, 2005.

7. Karlinsky, 2000, 2005.
} 
a abordés dans sa recherche sur la transformation du paysage britannique pendant les deux dernières décennies du Mandat ${ }^{8}$.

L'étude des techniques d'irrigation pendant le Mandat britannique demande de s'interroger sur les circulations de savoirs et les transferts techniques commencés à des rythmes et selon des temporalités différentes par la population arabe, les sionistes et les administrateurs britanniques. Les techniques agricoles développées par les Arabes sont héritières de leur présence multiséculaire en Palestine ainsi que des techniques nabatéennes, romaines et byzantines. Au XIx ${ }^{\mathrm{e}}$ siècle, selon Donald Quataert, l'importation par l'Empire ottoman de technologies développées en Occident dans tous les domaines de la société, notamment pendant la période des Tanzimat, apparaît comme nécessaire pour survivre à l'hégémonie occidentale 9 . Dans le cadre de la Palestine, à la fin du Xix ${ }^{\mathrm{e}}$ siècle, les transferts de technologies européennes ne sont pas uniquement décidés par la Sublime Porte mais se diffusent à travers la colonisation juive. De plus, cette dernière, au contact de la population locale, utilise et transforme les techniques développées par les Arabes. À ces transferts techniques suscités par les sionistes s'ajoute l'expertise britannique développée pendant le Mandat qui est elle-même nourrie de plusieurs décennies d'expériences coloniales variées au sein de l'Empire en matière de contrôle hydraulique et de techniques d'irrigation, en Inde en particulier ${ }^{10}$.

Dans cet article, nous examinerons les circulations de savoirs techniques liés à l'irrigation parmi la population arabe et les colons de la fin du XIX ${ }^{\mathrm{e}}$ siècle aux années du Mandat britannique (1922-1948). Nous étudierons ainsi les méthodes arabes de conservation et de pompage de l'eau et les apports techniques sionistes pendant cette période. Toutes ces techniques sont le fruit d'échanges technologiques tissés à l'échelle du Moyen-Orient et du monde sur un temps long.

\footnotetext{
8. El-Eini, 2006.

9. Quataert, 1992, p. 1.

10. Headrick, 1988, p. 171.
} 


\section{Les techniques arabes de pompage et de conservation de l'eau}

L'espace palestinien n'est pas homogène, les techniques agricoles et l'irrigation se développent en fonction des différentes caractéristiques physiques des régions qui le composent. Les méthodes de conservation, de pompage et de distribution de l'eau sont donc variées sur le territoire. L'irrigation est un procédé agricole en expansion puisque les cultures irriguées arabes augmentent de $37 \%$ entre 1930 et $1945^{11}$. Ce type de culture n'est cependant pas celui qui domine l'agriculture arabe. Celle-ci est davantage tournée vers les cultures sèches, telles que le blé et l'olive, peu gourmandes en eau comme l'a démontré Beshara Doumani dans son étude de la région de Naplouse au XIX ${ }^{\mathrm{e}}$ siècle $^{12}$.

Les techniques de maîtrise de l'eau en usage en Palestine à la fin du XIx ${ }^{\mathrm{e}}$ siècle et pendant le Mandat sont héritières des évolutions des techniques hydrauliciennes arabes depuis l'époque médiévale. Comme l'a montré l'historien de l'agronomie Mohammed El Faïz dans sa recherche sur l'hydraulique arabe $^{13}$, le monde arabe a connu d'importants développements techniques pour le captage des eaux du ciel et souterraines et le contrôle des eaux de surface de la période préislamique aux premières intrusions européennes contemporaines.

\section{Processus de conservation de l'eau et adaptation à la rareté de la ressource hydrique dans les villages arabes de Palestine}

La ressource hydrique détermine les potentialités et les orientations agricoles d'un territoire tout autant que la qualité de la terre ou le climat. La lecture des rapports écrits par les agents de renseignement de la Haganah ${ }^{14}$ sur les villages arabes de Palestine à la fin de la Seconde Guerre mondiale permet de prendre la mesure de l'inégalité de la répartition des ressources hydriques et des difficultés rencontrées par les habitants pour l'approvi-

11. Nadan, 2006.

12. Doumani, 1995.

13. El Faïz, 2005.

14. La Haganah est la force de défense juive fondée en 1920. 
sionnement en eau tant pour l'agriculture que pour la vie domestique. Chaque note contient en effet une description des ressources en eau à disposition de la population locale et laisse entrevoir les techniques utilisées pour la conservation de l'eau. Ainsi, le village de Saris, près de Jérusalem, n'a pour ressources en eau que des réservoirs de collecte de l'eau de pluie qui s'assèchent pendant les années arides. La situation est identique dans le village de Jaljulia près de Kfar Saba ainsi que dans de très nombreux villages de Palestine qui ne disposent ni de ressources d'eau souterraine, ni de cours d'eau à proximité ${ }^{15}$. Ce manque d'eau a pour conséquence le développement de techniques agricoles particulières et de stratégies de conservation des eaux de pluies.

Dans les montagnes du centre de la Palestine, nommées à l'époque Monts de Judée, la culture en terrasses résulte de la prise en compte par les hommes de la combinaison des facteurs topographiques, climatiques et hydrographiques depuis l'Antiquité ${ }^{16}$. Ces montagnes sont calcaires et disposent de peu de cours d'eau et de ressources souterraines, mais connaissent une pluviométrie conséquente entre octobre et avril. La construction de plusieurs étages le long de la pente de la montagne permettait une meilleure infiltration des eaux de pluies et l'atténuation des phénomènes d'inondation lors de fortes précipitations. Chaque terrasse est ainsi fermée par un muret construit avec les pierres collectées dans les environs par les paysans afin d'éviter tout processus d'érosion. La culture en terrasses n'est absolument pas propre à la Palestine : ce procédé agricole est utilisé de la Chine au Pérou en passant par le Liban voisin ${ }^{17}$. Dans le district de Ramallah, les oliviers et les figuiers sont plantés dans des trous communs sur les terrasses. Ces cultures destinées à la consommation locale sont produites grâce à ce mode de gestion du sol et des eaux de pluies ${ }^{18}$.

La conservation des eaux de pluies dans des citernes est primordiale dans l'espace rural palestinien pour assurer la vie des paysans et des cultures. Les réservoirs des eaux de pluies sont construits le plus souvent en pierres et la plupart de ceux qu'utilisent les fellahin pendant le Mandat datent de l'Antiquité. Le désert du Néguev est un espace intéressant pour l'étude des différents procédés de conservation des eaux de pluies et des sources

15. Archives de la Haganah, Dossier א/95/105, 1947.

16. Amiran, 1963, p. 696.

17. Lewis, 1953.

18. Keren Kayémeth Léisrael, 1928, p. 342. 
souterraines. En l'absence de cours d'eau et de ressources souterraines pérennes, les habitants du désert ont développé des techniques particulières, adaptées à l'aridité et à la topographie du terrain, pour assurer leur survie et celle de leurs troupeaux. Une des plus anciennes techniques d'approvisionnement en eau est le qanat, un système de tunnels de récupération de l'eau reliés à une série de puits en surface. Des traces archéologiques permettent de faire remonter ce procédé à l'Iran du I ${ }^{\text {er }}$ millénaire avant notre ère ${ }^{19}$. Pendant le Mandat, les qanats du Néguev, situés dans la vallée de l'Arava, sont tombés en désuétude ${ }^{20}$. Les réservoirs d'eau de pluie sont les principaux points d'approvisionnement pour les Bédouins du désert aux $\mathrm{XIX}^{\mathrm{e}}$ et $\mathrm{XX}^{\mathrm{e}}$ siècles. Ainsi, des citernes creusées à même la roche, situées en contrebas des pentes, récupèrent l'eau de ruissellement. Elles sont surmontées d'un toit en pierre afin de limiter l'évaporation. Certaines de ces citernes datent des Nabatéens ${ }^{21}$. Il y en a des centaines de ce type dans le Néguev. De plus, l'eau des wadis est conservée dans des réservoirs de pierres car ces cours d'eau non-pérennes s'assèchent pour la plupart complètement hors de la saison des pluies.

Les techniques de conservation des eaux de pluies sont nées des échanges de savoirs entre les sociétés de l'Orient antique, elles ont peu évolué jusqu’à l'arrivée des Britanniques en Palestine.

\section{Les méthodes traditionnelles de pompage de l'eau pour l'irrigation}

Les méthodes traditionnelles pour puiser l'eau dans une source souterraine en Palestine sont héritières d'un ensemble de circulations techniques depuis plusieurs siècles, voire millénaires. En effet, les procédés de pompage de l'eau par force motrice humaine ou animale dont la présence de certains, comme le shadoof, est attestée par l'archéologie depuis le III ${ }^{\mathrm{e}}$ millénaire avant notre ère, sont toujours en usage en Palestine ottomane du $\mathrm{XIX}^{\mathrm{e}}$ siècle et pendant le Mandat ${ }^{22}$.

19. Sur les qanats: Globot, 1979 et Beaumont, Bonine et McLachlan (dir.), 1989.

20. Evanari, Shanan et Tadmor, 1971, p. 173.

21. Evanari, Shanan et Tadmor, 1971, p. 159.

22. Béthemont, 1981, p. 10. 
La technique du shadoof pour pomper l'eau des puits est une des plus répandues en Palestine et plus généralement au Moyen-Orient au XIX ${ }^{\mathrm{e}}$ siècle $^{23}$. Le shadoof est un système de levier à bascule : un seau en bois ou en terre cuite est placé au bout d'un balancier actionné par un homme. Développée depuis le $\mathrm{II}^{\mathrm{e}}$ millénaire avant notre ère en Égypte ${ }^{24}$, cette méthode ne permet pas une irrigation de grande ampleur. Par conséquent, d'autres techniques de pompage de l'eau pour l'irrigation cohabitent avec le shadoof.

À la lecture des écrits de l'archéologue Edward Robinson, spécialiste de la Terre Sainte, publiés au XIX ${ }^{\mathrm{e}}$ siècle, nous pouvons voir émerger différentes techniques de pompage de l'eau'. Dans son ouvrage intitulé Physical Geography of the Holy Land, publié à titre posthume, il décrit les villages qu'il a traversés et apporte de précieuses informations sur les techniques d'irrigation employées :

Le mode de prélèvement de l'eau est varié. Lorsque le puits n'est pas trop profond, la corde du seau est généralement descendue et tirée à la main. À Summeil, quand le puits a une profondeur de plus de cent pieds, la corde est passée au-dessus d'un puits ; et plusieurs femmes la tirent en parcourant une longue distance dans les champs. Pour les puits encore plus profonds de Hûj et Dâniyâl, la corde était fixée de la même manière sur poulie, et était tirée par une paire de bœufs qui s'éloignait en ligne droite dans les champs. À Sukkarîyeh, un village qui vient d'être reconstruit par le Sheikh Sa'îd, gouverneur de Gaza, une Sâkieh égyptienne a été introduite ; c'est une longue corde, avec des jarres attachées à celle-ci, passant sur une roue au-dessus du puits, qui tourne par la force d'un chameau tournant en cercle $^{26}$.

Les exemples présentés par Edward Robinson sont tous localisés dans la région de Gaza, c'est-à-dire le long de la plaine côtière. Dans cette région, l'eau pour l'irrigation est principalement issue de ressources souterraines. Les différentes techniques développées par la population arabe locale sont adaptées à la profondeur des puits. La technique la plus simple de remontée des eaux d'un puits consiste à y plonger un seau et le hisser en tirant sur

23. Robinson, 1865, p. 247.

24. Viollet, 2005, p. 15.

25. Sur l'œuvre d'Edward Robinson : Soler, 2014.

26. Robinson, 1865, p. 247 (traduction E. M.). 
une corde. Cette technique est loin d'être propre au Moyen-Orient mais elle est toujours en usage en Palestine pendant la période mandataire comme l'atteste une photographie anonyme prise pendant la première moitié du $\mathrm{xx}^{\mathrm{e}}$ siècle (Fig. 1). Sur cette photographie, l'eau est remontée du puits par les Bédouins grâce à des cordes pour être ensuite conservée dans des jarres en terre cuite. Les hommes prélèvent ici l'eau par la seule force de leurs bras. Dans les cas d'un puits plus profond, la puissance motrice des animaux de trait comme l'âne, le bœuf ou le chameau est sollicitée. La technique la plus simple consiste alors à relier par une corde des animaux de trait à une poulie fixée par une armature en bois au-dessus du puits. Par sa force de traction, l'animal remonte ainsi la jarre en terre cuite remplie d'eau du fond du puits. C'est le deuxième mode de pompage de l'eau décrit par Edward Robinson dans la région de Gaza. Cette méthode est attestée également pendant le Mandat comme le montre cette photographie prise entre 1920 et 1933 en Palestine (Fig. 2). Là, ce sont deux bovins menés par un paysan qui remontent l'eau prélevée d'un puits en pierre. Selon Edward Robinson, une méthode plus complexe de prélèvement de l'eau des puits a été introduite dans la région de Gaza autour des années 1850 : la Sakia. Il s'agit d'une machine hydraulique généralement nommée en anglais « roue orientale » et en français " roue égyptienne » ou " roue persane ». Son importance pour l'irrigation à partir de ressources hydriques souterraines est indiquée dans la plupart des travaux sionistes sur l'agriculture en Palestine, comme le livret d'Isaac Elazari-Volcani intitule The Fellah Farm ${ }^{27}$ ou encore le Report of the experts submitted to the Joint Palestine Survey Commission publié en 1928. La sakia incarne pour les sionistes la "méthode arabe de pompage de l'eau ${ }^{28}$ ", c'est-à-dire une technique traditionnelle opposée aux nouvelles techniques de pompage motorisé répandues au cours du Mandat dans les colonies agricoles juives. La construction de cette machine hydraulique est coûteuse : il s'agit d'une structure en bois avec une roue verticale munie d'une chaîne à godets en terre cuite ou en bois actionnée par une roue horizontale, ellemême reliée à une barre en bois ou à une corde qui permet à l'ensemble de se mettre en branle grâce à la force d'un animal de trait tournant en cercle ${ }^{29}$. Les godets remplis d'eau sont ensuite déversés dans les rigoles d'irrigation creusées dans le sol.

27. Elazari-Volcani, 1930, p. 1.

28. Keren Kayémeth Léisrael, 1928, p. 132.

29. Menassa, Laferrière, 1974. 


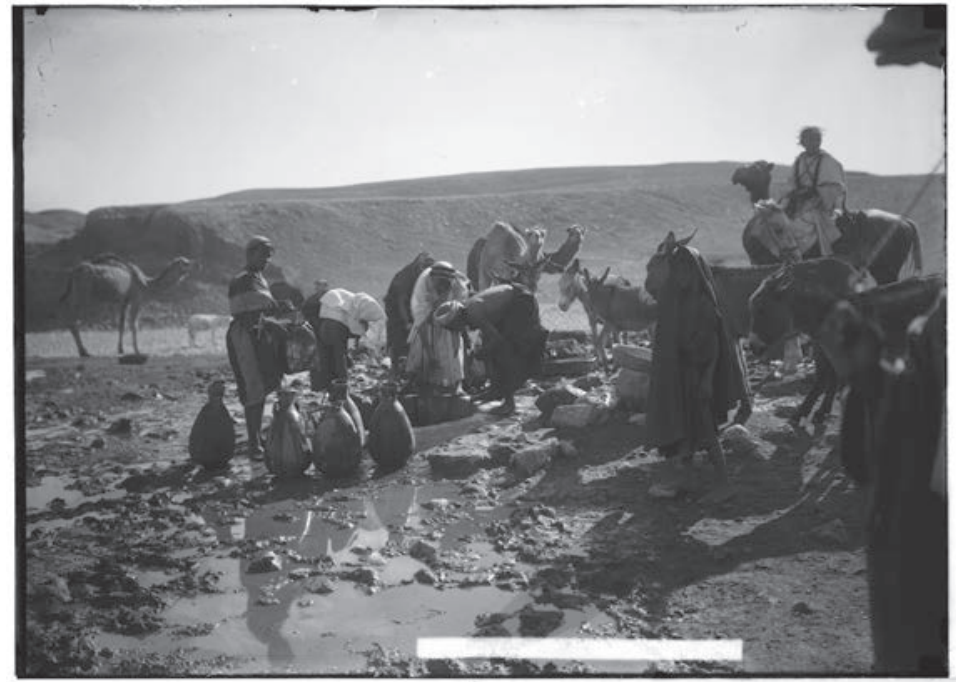

Fig. 1. - La collecte de l'eau dans un puits du Néguev pendant la première moitié du $\mathrm{xx}^{\mathrm{e}}$ siècle

Matson Photograph Collection, Library of Congress Prints and

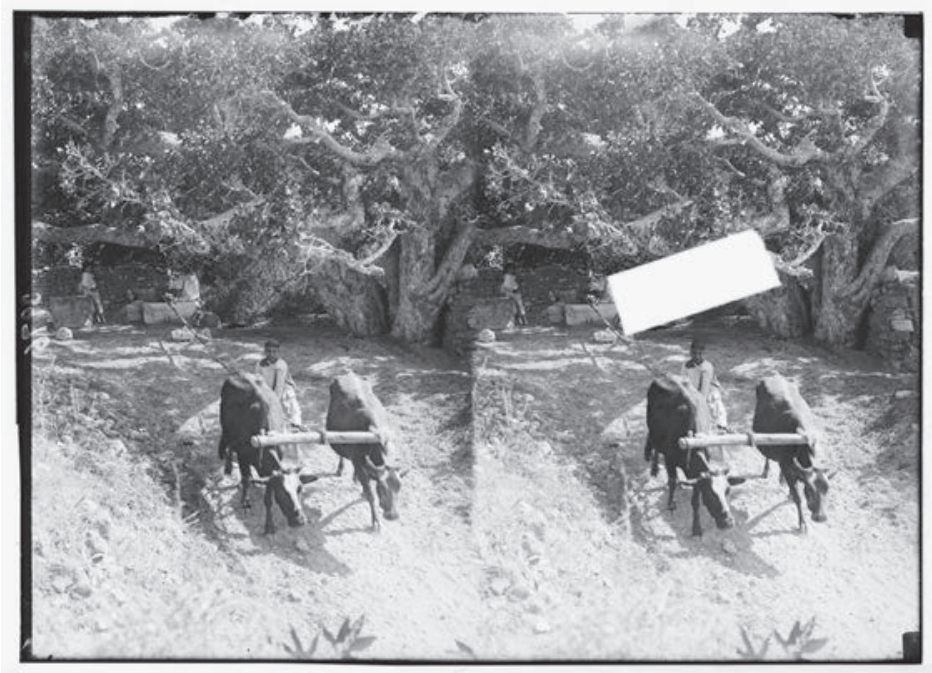

Fig. 2. - Le puisage de l'eau par la force motrice de bovins en Palestine pendant la période mandataire

Matson Photograph Collection, Library of Congress Prints and Photographs Division, Washington, États-Unis, LC-M32- 3976 


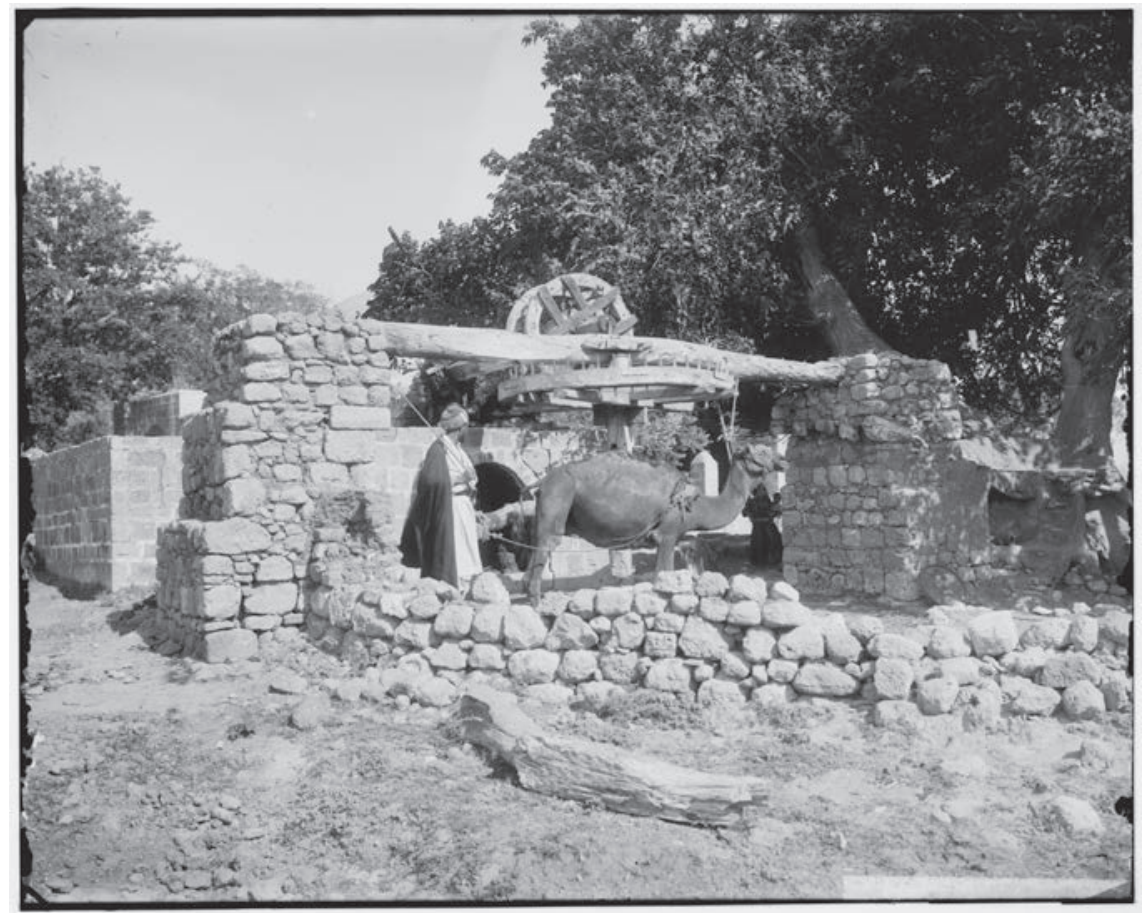

Fig. 3. - La sakia en Palestine, première moitié du xx siècle

Matson Photograph Collection, Library of Congress Prints and Photographs

Division, Washington, États-Unis, LC-M36- 1151

Un seul regard sur cette photographie datant de la première moitié du $\mathrm{xx}^{\mathrm{e}}$ siècle (Fig. 3) permet de deviner le coût considérable de cette machine élévatrice : la fabrication des roues en bois demande un travail de menuiserie conséquent, l'ensemble de la structure du puits est entouré de briques tandis que les animaux de traits renforcent le prix de cet investissement pour l'irrigation des terres de Palestine. La capacité de ce type de roue élévatrice oscille entre cinq et huit mètres cubes d'eau par heure et la profondeur atteinte est d'un maximum de douze mètres ${ }^{30}$. La sakia, pour Edward Robinson, viendrait de l'Égypte voisine. Cette technique s'est effectivement largement diffusée en Égypte et répandue au sein de l'Empire Ottoman. Le berceau de ce procédé d'élévation de l'eau n'est pourtant pas le MoyenOrient puisqu'au $\mathrm{II}^{\mathrm{e}}$ siècle avant notre ère, les traces d'une roue élévatrice à godets ont été retrouvées en Italie centrale ${ }^{31}$.

30. Karlinsky, 2005, p. 93.

31. Viollet, 2005, p. 19. 
Pendant le Mandat, il convient d'associer les transferts techniques en Palestine et les capacités financières des différents groupes sociaux. Comme l'a démontré Amos Nadan, il est en effet difficile pour un fellah de se munir de systèmes d'irrigation similaires à ceux construits dans les colonies juives en raison de ses plus faibles capacités d'investissement financier ${ }^{32}$. Les sionistes construisent progressivement une représentation négative des techniques agricoles arabes responsables, selon eux, de la faiblesse des rendements agricoles et de la destruction de l'environnement palestinien. En 1928, le rapport d'experts envoyé par l'Organisation sioniste décrit ainsi la Palestine : "Jadis fortement peuplé dans ses espaces les plus fertiles, jadis patrie d'une agriculture prospère, même si primitive, c'est aujourd'hui un pays pauvre et peu développé. Les terrasses des montagnes et le sol se sont effondrés. La fertilité de ses vallées a été compromise par des siècles de culture inefficace ${ }^{33}$ ». Ce discours, très commun à l'époque mandataire, cherche à délégitimer l'agriculture arabe afin de souligner, par contraste, la modernité des procédés agricoles utilisés dans les colonies sionistes.

\section{Les transferts de techniques agricoles en Palestine et la colonisation agricole sioniste}

L'établissement par les premiers colons juifs de villages agricoles, notamment avec la création de la première ferme-école juive Mikveh-Israël en 1870 par l'Alliance israélite universelle, contribue progressivement au transfert de techniques agricoles européennes en Palestine. Dans la décennie suivante, les pogroms dont les Juifs d'Europe de l'Est sont victimes provoquent le départ d'une partie des populations juives vers l'Europe occidentale, mais également vers la Palestine ottomane ${ }^{34}$. Le groupe des Amants de Sion, Hovevei Sion, constitué en Europe de l'Est, fonde ainsi un premier village agricole en 1882 : Rishon-le-Tsion. Ce mouvement agricole juif en Terre Sainte est bientôt soutenu par le baron français Edmond de Rothschild qui finance les colonies agricoles ${ }^{35}$ et les encadre au sein de deux organisations qui se succèdent : la Jewish Colonization Association à

32. Nadan, 2006, p. 64.

33. Kéren Kayémeth Léisrael, 1928, p. 17 (traduction E. M.).

34. Laurens, 2007, p. 102.

35. Aharonson, 2000. 
partir de 1899, remplacée par la Palestine Jewish Colonization Association en 1924. Parallèlement, la fin du $\mathrm{XIx}^{\mathrm{e}}$ siècle est le temps de l'émergence du sionisme politique avec la tenue du premier congrès sioniste mené par Théodore Herzl à Bâle en 1897. Le sionisme est la recherche d'un territoire souverain pour le peuple juif en diaspora. Exclusivement tourné vers la Palestine ottomane depuis les premières années du $\mathrm{xx}^{\mathrm{e}}$ siècle ${ }^{36}$, le dessein sioniste porte en lui le projet agricole de retour des Juifs au travail de la terre $^{37}$. Pour soutenir le développement de l'agriculture et de la colonisation, l'Organisation sioniste crée le Fonds national juif (Keren Kayemeth Leisrael) en 1901, chargé de l'achat de terres pour les Juifs, et l'organisme financier sioniste le Keren Hayessod.

Depuis la fin du XIX ${ }^{\mathrm{e}}$ siècle, l'expansion des cultures irriguées par les colons juifs et sionistes s'est accompagnée de l'introduction de techniques agricoles venues d'Europe et d'Amérique du Nord. Derek Penslar a démontré dans son travail sur les ingénieurs de la colonisation juive ${ }^{38}$ comment les techniciens français liés à l'œuvre philanthropique du baron de Rothschild ont importé leurs connaissances agronomiques et techniques en Palestine. Puis, dans les premières années du $\mathrm{Xx}^{\mathrm{e}}$ siècle, l'expertise agricole allemande et russe prend progressivement le pas sur la française. De plus, la colonisation juive, au contact de la population locale, utilise et transforme les techniques développées par les Arabes. Les sources à notre disposition pour analyser les techniques d'irrigation sionistes proviennent principalement des archives des principales institutions sionistes. Loin de représenter la totalité des implantations agricoles juives en Palestine, les évolutions techniques perceptibles dans les archives sionistes n'en démontrent pas moins les changements agricoles de la période pré-mandataire et mandataire.

Le développement de l'irrigation revêt un caractère éminemment politique pour les sionistes. En effet, l'augmentation des rendements agricoles est indispensable pour permettre l'immigration massive de Juifs du monde entier vers la Palestine. Le Fonds national juif et les organisations agricoles juives tentent d'accroître la capacité d'absorption économique du pays pour faire venir de nouveaux migrants juifs. Enjeu politique majeur à une période où les Britanniques souhaitent limiter l'immigration juive en

36. Charbit, 2011, p. 21.

37. Bensoussan, 2005.

38. Penslar, 1991. 
fonction de la capacité d'absorption économique. C'est par l'importation de nouvelles techniques agricoles et par la transformation des procédés agricoles que les sionistes essaient d'atteindre cet objectif comme l'affirme en 1925 Arthur Ruppin, chargé des questions foncières pour l'Organisation sioniste : "Les premières étapes du mouvement national juif ont montré que l'agriculture doit être le fondement économique de l'immigration juive en Palestine ${ }^{39}$ ».

\section{L'appropriation et la transformation des techniques agricoles arabes}

Les colonies juives se sont progressivement approprié l'agrumiculture de la plaine côtière. Le modèle californien d'irrigation est importé au cours de la période mandataire afin d'accroître le rendement agricole et passer à une agriculture intensive. Comme l'a montré Nahum Karlinsky dans sa recherche sur l'agrumiculture ${ }^{40}$, les sionistes tournent leur regard vers l'agriculture californienne dont les caractéristiques physiques et climatiques sont assez similaires à celles de la plaine côtière. Ainsi, l'Organisation sioniste fait venir à deux reprises le professeur d'agronomie, Elwood Mead, spécialiste de l'irrigation en Californie et en Australie : en 1923 et en $1927^{41}$. Il visite la Palestine rurale pendant plusieurs semaines ; puis, il fait part à l'exécutif sioniste de son avis sur les potentialités agricoles du pays. Dans son rapport écrit à la suite de sa première visite en 1924, le professeur de l'université de Berkeley incite les sionistes à accroître les surfaces de cultures irriguées pour passer à un modèle d'agriculture inten$s_{i v e}{ }^{42}$. L'introduction de pompes à moteur pour pomper l'eau est un des premiers changements majeurs qui entraîne l'amélioration du rendement agricole. Les pompes à moteur fonctionnent au diesel et ont une puissance moyenne de douze chevaux. Celles-ci sont placées au fond du puits ${ }^{43}$. Une vingtaine d'années plus tard, en 1943, le rapport du fonctionnaire

39. Ruppin, 1926, p. 1 (traduction E. M.).

40. Karlinsky, 2005.

41. Teisch, 2011.

42. Central Zionist Archives, S25/658/10, Elwood Mead, Report to Zionist Executive, London, on Agricultural Development in Palestine, 1924.

43. Keren Kayémeth Léisrael, 1928, p. 273. 
britannique F. C. Carver sur les pompes à moteur au Moyen-Orient souligne le haut développement de cette technologie en Palestine ${ }^{44}$.

Les agriculteurs juifs remplacent progressivement les cuvettes et les sillons creusés à même la terre par des systèmes de canaux d'irrigation en métal. Ces investissements, coûteux, sont financés notamment par des associations de kibboutzim, l'Organisation sioniste et la Palestine Jewish Colonization Association. Dans les années 1930, l'irrigation par aspersion se développe dans les terres agricoles exploitées par les sionistes. Publié en 1941, le rapport conjoint de divers comités juifs comme l'organisation des producteurs de légumes et le syndicat des travailleurs agricoles donne des éléments précieux sur les techniques d'irrigation par aspersion utilisées et testées depuis une décennie ${ }^{45}$. Deux principaux types d'irrigation par aspersion sont installés dans les mochavim et les kibboutzim : un système mobile et un autre fixe, tous les deux composés de canaux connectés à des arroseurs rotatifs ou non-rotatifs. La spécificité du système mobile est simple : les canaux, les fixations et l'arroseur peuvent être retirés facilement pour permettre le labourage de la terre par des machines mécaniques. Les arroseurs sont placés sur les canaux tous les 14 à 16 mètres. Le rapport des organisations agricoles sionistes de 1941 énumère tous les avantages et les inconvénients des systèmes d'aspersion installés en Palestine depuis une décennie. L'arroseur rotatif semble avoir leur faveur en raison de sa capacité à fournir de l'eau de manière égale sur l'ensemble d'une parcelle. Depuis les années 1930, ces systèmes d'irrigation par aspersion, que ce soit les robinets, les canaux, les arroseurs, sont construits par des sociétés juives en Palestine comme Sqiner ou Metar ${ }^{46}$.

Pendant le Mandat, les sionistes ont profondément transformé les procédés de collecte des eaux. Les techniques nabatéennes, byzantines et arabes de collecte des eaux de pluies par gravité grâce à l'installation de réservoirs à flanc de pente ${ }^{47}$ dans le désert du Néguev sont utilisées par les sionistes qui cherchent à les perfectionner pour permettre l'irrigation des terres qui entourent les colonies juives construites dans cette région

44. « Pump irrigation form wells has already been brought to a high pitch of development in Palestine ", Midde East Center Archives, GB165-0203, Box 26, Carver F.C., Report on Pump Irrigation in the Middle East, 6 août 1943.

45. CZA, S90/2123/2, "Saqira al hahamtara bemakshei haovdim", 31 décembre 1941.

46. Ibid.

47. Evanari, Shanan, Tadmor, 1982, p. 159. 
dans les années 1940. Le début de l'année 1946 voit l'inauguration des infrastructures hydrauliques de collecte des eaux de pluies pour irriguer les terres agricoles de la colonie juive Revivim, située au nord du Néguev. L'eau, à la saison des pluies, ruisselait sur les pentes pour rejoindre le Wadi Aslouj en contrebas : l'objectif des sionistes est alors de canaliser cette eau pour en faire la principale source d'alimentation des cultures agricoles. Financée par le Fonds national juif et l'Agence juive, un barrage en béton armé est bâti pour contenir l'eau qui coule dans le Wadi Aslouj ${ }^{48}$. L'eau est ensuite amenée vers les terres irriguées de Revivim par un canal de 800 mètres de long vers un réservoir souterrain lui aussi en béton. Ce réservoir peut contenir 220000 mètres cubes, le canal ayant une capacité d'approvisionnement de $10000 \mathrm{~m}^{3}$ par heure.

Les sionistes investissent largement dans la recherche agronomique pour permettre ces mutations techniques. Ils créent progressivement des technologies capables de contourner le manque d'eau ou les difficultés topographiques pour permettre le développement économique et agricole du territoire palestinien dans le but de servir leur dessein politique.

\section{Les recherches et les techniques au service de l'immigration juive}

Dès 1921, l'Exécutif sioniste crée la première station agricole expérimentale de Ben Shemen, déplacée dix ans plus tard à Rehovot. Cette station est le premier institut scientifique d'expérimentation agricole sioniste fondé en Palestine : elle mène des recherches agricoles pour améliorer les cultures des villages juifs. Les résultats de ses recherches sont publiés sous forme de livrets. Avant la mise en place du Mandat britannique, la ferme-école Mikveh-Israël, fondée en 1870 au sud de Jaffa, faisait office de laboratoire d'expérimentation agricole pour répondre aux difficultés rencontrées par les premiers pionniers juifs. Les domaines de recherches agricoles sont très variés : entomologie, zoologie, agronomie, botanique, chimie du sol, géologie et hydrologie. De nombreuses expériences sont conduites pour améliorer le modèle agricole juif. Ainsi, les agronomes et leaders sionistes Selig

48.« Negev rain water for irrigation », Palestine Post, 7 janvier 1946. 
Soskin ${ }^{49}$, Isaac Elazari-Volcani ${ }^{50}$ et Moshe Smilansky ${ }^{51}$ ont tous trois publié des travaux incitant les colons juifs à adopter un modèle agricole intensif fondé sur l'expansion de la culture irriguée.

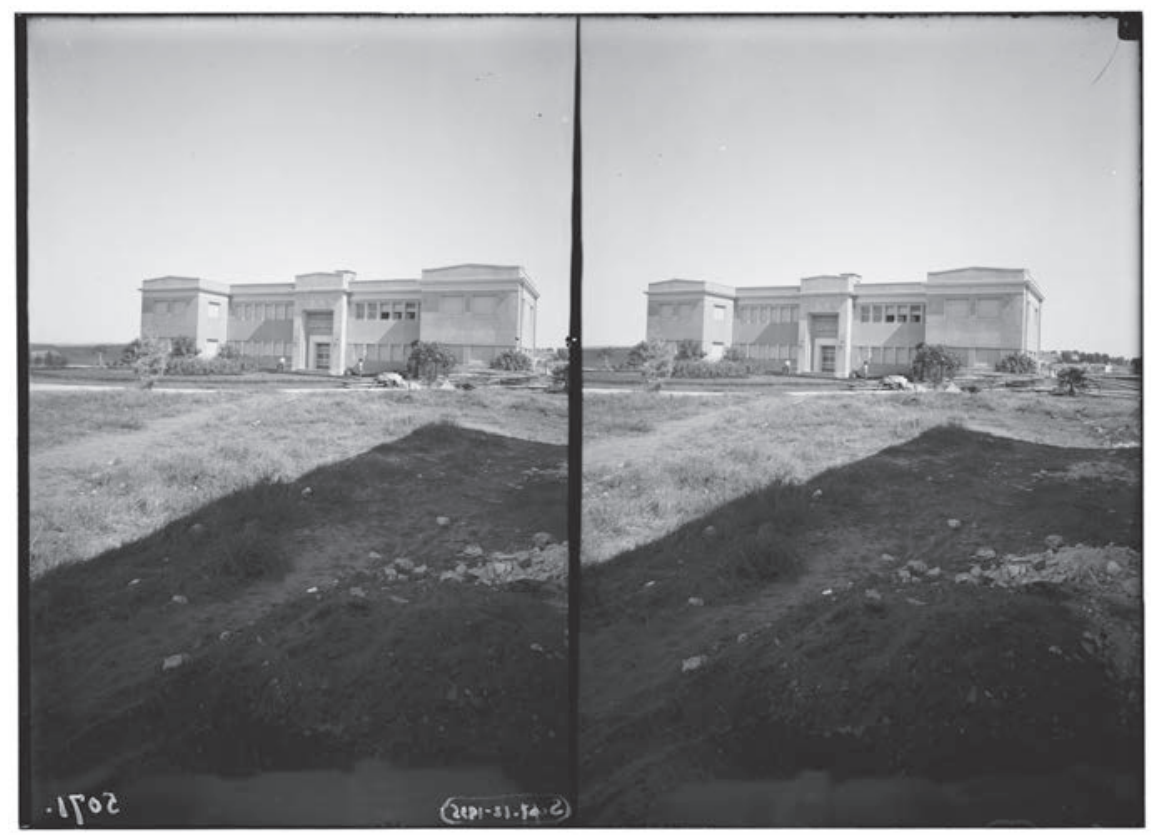

Fig. 4. - La station agricole expérimentale de Rehovot en 1935

Matson Photograph Collection, Library of Congress Prints and Photographs Division, Washington, États-Unis, LC-M32- 5071

Les chercheurs de la Station expérimentale de Rehovot (Fig. 4), affiliés à l'université hébraïque de Jérusalem, travaillent également à résoudre les problèmes rencontrés quotidiennement par les colons juifs dans leurs fermes. C'est le cas d'Adolf Reifenberg, biochimiste allemand né en Allemagne en 1899, arrivé en Palestine avant la Première Guerre mondiale, qui a consacré sa recherche aux propriétés physiques des sols irrigués ${ }^{52}$. De la même manière, Fritz Shimon Bodenheimer, entomologiste formé en Allemagne, rejoint les rangs de la Station expérimentale de Rehovot et de

49. Soskin, s.d.

50. Elazari-Volcani, 1935.

51. Smelansky, 1930.

52. Reifenberg, 1935. 
l'université hébraïque pendant le Mandat afin de travailler sur les insectes nuisibles aux cultures irriguées ${ }^{53}$.

À partir des années 1930, les sionistes s'intéressent à l'utilisation de l'eau salée pour l'agriculture. Le développement de l'irrigation dans la plaine côtière a eu, à certains endroits, des conséquences négatives sur la qualité des eaux souterraines. En 1947, cette situation est soulignée dans le rapport du Gouvernement de Palestine sur les ressources hydriques : "Près de Tel-Aviv, le pompage excessif a entraîné l'intrusion d'eau de mer dans plusieurs puits ; dans les dunes de Haïfa, d'autres puits sont menacés ${ }^{54}$ ". Dès les années 1940, les sionistes souhaitent s'implanter dans la région désertique du Néguev où beaucoup des ressources souterraines ont un taux de sel très élevé ${ }^{5}$. Dans le but de permettre l'accroissement des cultures irriguées, les sionistes cherchent à trouver des moyens techniques pour contourner le problème posé par les ressources d'eau salée. Pour cela, le docteur A. Werber a mené une série d'expérimentations pour déterminer si le sol pouvait absorber sans conséquence une eau avec un taux de chlore important et si des techniques pouvaient être mises en place pour retirer le sel infiltré dans la terre ${ }^{56}$. En janvier 1948, un article du quotidien Haaretz annonce également l'invention d'une machine capable de retirer le sel de l'eau pour produire de l'eau potable dans le $\mathrm{Néguev}^{57}$. Cette nouvelle technologie a été développée par le centre de recherche de Rehovot et par des chercheurs états-uniens.

\section{Conclusion}

Indéniablement, la période du Mandat britannique est celle d'importantes mutations des techniques d'irrigation. Le pompage motorisé, les nouveaux types de canaux d'irrigation ne sont pas uniquement développés en Palestine mais également dans l'ensemble du Moyen-Orient et de l'Afrique du Nord. Néanmoins, la particularité de la situation palestinienne réside

53. Bodenheimer, 1925.

54. Government of Palestine, 1947, p. 6.

55. MECA, Sir Cunningham Collection, private papers. Box 6. "Visit of H.E. the High Commissioner to Beth Eshel, Beesheba Sub-District on Thursday 29th of November", 1945.

56. Werber, 1936.

57. CZA, S90/2123/2, "Houmtza makhchir leafqat mi chtia mimei-melakh banegev ", Haaretz, 16 janvier 1948. 
dans le fait que les transferts technologiques sont lancés non par une puissance coloniale mais par l'Organisation sioniste et ses ramifications en Palestine. Les technologies sont au cœur du discours politique forgé par les sionistes pour délégitimer la présence arabe en Palestine et fustiger l'ancienne domination ottomane. Ainsi, Arthur Ruppin au milieu des années 1930 affirme-t-il que "Pendant les quatre siècles de domination ottomane, la Palestine fut tristement négligée. On a laissé les merveilleuses infrastructures hydrauliques romaines se dégrader, les forêts ont été coupées, tandis que sous le système féodal le sol était cultivé de manière irrationnelle ${ }^{58}$ ". Les sionistes se positionnent ainsi comme les porteurs de la modernité technique face à ce qu'ils perçoivent comme des procédés primitifs. Ils nient ainsi le temps long et les échanges de connaissances dans lesquels s'inscrivent les techniques d'irrigation arabes.

\section{Sources et bibliographie}

\section{Archives}

CZA, S90/2123/2, "Houmtza makhchir leafqat mi chtia mimei-melakh banegev ", Haaretz, 16 janvier 1948.

CZA, S90/2123/2, "Negev rain water for irrigation ", Palestine Post, 7 janvier 1946.

CZA, S90/2123/2, "Saqira al hahamtara bemakshei haovdim ", 31 décembre 1941.

MECA, Sir Cunningham Collection, private papers. Box 6, "Visit of H.E. the High Commissioner to Beth Eshel, Beesheba Sub-District ", 29 novembre 1945.

MECA, GB165-0203, Box 26, Carver, F.C., Report on Pump Irrigation in the Middle East, 6 août 1943.

\section{Sources imprimées}

BodenheImer Shimon Fritz, The Mediterranean fruit fly in Palestine, The Zionist

Organisation, Institute of Agriculture and Natural History, publié avec la coopération de l'association Pardes, janvier 1925.

Elazari-Volcani Isaac, The Fellah's farm, The Jewish Agency for Palestine, TelAviv, 1930.

Elazari-Volcani Isaac, Rational Planning of Agricultural Settlement in Palestine, Karen Hayesod, Jérusalem, 1935. 
Keren Kayemeth Leisrael, Reports of the experts submitted to the Joint Palestine survey commission, 1928.

Smelansky M., Jewish Colonisation and the Fellah. The Effect of Jewish Land Settlement in Palestine on the Native Rural Population and on Agricultural Development in general, The Mischar w'Taasia Publishing Co. Ltd, Tel-Aviv, 1930.

Soskin Selig, Dr., Land settlement in Palestine, some ideas on intensive cultivation and close settlement for Palestine, Printed for Private Circulation, Printing in Great Britain by Billing and Sons, s.d.

Reifenberg Adolf, A. Irrigation damages in Citrus Plantation, Hebrew University, Hadar, décembre 1935, vol. VIII n 12.

Robinson Edward, Physical Geography of the Holy Land, John Murray, Londres/ Crocker and Brewster, Boston, 1865.

Gottmann Jean, "L'irrigation en Palestine », Annales de Géographie, t. 4, n² 248 , 1935 , p. 143-161.

Government of Palestine, Memorandum on the Water Resources of Palestine, presented by the Government of Palestine to the United Nations' Special Committee on Palestine in July, 1947. Jerusalem, Goverment Printer, Government of Palestine.

Ruppin Arthur, The Agricultural Colonisation of the Zionist Organisation in Palestine, Martin Hopkinson and Company Ltd, Londres, traduit de l'allemand par R. J. Feiwel, 1926.

\section{Bibliographie}

Aharonson Ran, Rothschild and early Jewish colonization in Palestine, The Hebrew University Magnes Press, Jérusalem, 2000.

Amiran David H. K, "L'utilisation du sol en Israël ", Annales de Géographie, t. $72, n^{\circ} 394,1963$, p. 693-719.

Beaumont Peter, Bonine Michael, McLachlan Keith (dir.), Qanat, kariz and khattara: traditional water systems in the Middle East and North Africa, Middle East Centre, School of Oriental and African Studies, University of London in Association with Middle East and North African Studies Press, Wisbech, 1989.

Bensoussan Georges, Une histoire intellectuelle et politique du sionisme 18601940, Fayard, Paris, 2005.

Bethemont Jean, "Sur les origines de l'agriculture hydraulique ", dans Metral Jean, Sanlaville Paul (dir.), L'Homme et l'eau en Méditerranée et au Proche Orient, GIS-Maison de l'Orient, Presses universitaires de Lyon, Lyon, 1981, vol. 4.

Charbit Denis, "Chronologie du sionisme et de l'État d'Israël », Cités, n 47-48, 2011, p. 19-26. 
Doumani Beshara, Rediscovering Palestine, Merchants and Peasants in Jabal Nablus, 1700-1900, University of California Press, Berkeley, 1995.

EL-EIni Roza, Mandated Landscape: British Imperial rule in Palestine, 1929-1948, Routledge, London, 2006.

EL FAïz Mohammed, Les maîtres de l'eau. Histoire de l'hydraulique arabe, Actes Sud, Arles, 2005.

Evanari Michael, Shanan Leslie, Tadmor Naphtali, The Negev. The Challenge of a Desert, Harvard University Press, Cambridge, 1971.

GLовот Henri, Les qanats. Une technique d'acquisition de l'eau, Mouton, Paris, 1979.

Headrick Daniel R., The Tentacles of Progress. Technology Transfer in the Age of Imperialism, 1850-1940, Oxford University Press, Oxford, 1988.

Kamen Charles S., Little Common Ground: Arab Agriculture and Jewish Settlement in Palestine, 1920-1948, University of Pittsburgh Press, Pittsburgh, 1991.

Karlinsky Nahum, "California dreaming: Adapting the "California Model" to the Jewish Citrus Industry in Palestine, 1917-1939», Israel Studies, 2000, vol. $5, \mathrm{n}^{\circ} 1$, p. 24-40.

KarLinsky Nahum, California dreaming: ideology, society, and technology in the citrus industry of Palestine, 1890-1939, State University of New York Press, New York, 2005.

Laurens Henry, La question de Palestine, Tome premier : 1799-1922, linvention de la Terre sainte, Fayard, Paris, 2007.

Levine Mark, Overthrowing Geography: Jaffa, Tel-Aviv, and the Struggle for Palestine, 1880-1948, University of California Press, Berkeley, 2005.

LewIs Norman N., "Lebanon. The Mountain and Its Terraces ", Geographical Review, vol. 43, n 1, janvier 1953, p. 1-14.

Menassa Laila, Laferriere Pierre, La Saqia, technique et vocabulaire de la roue à eau égyptienne, Institut français d'archéologie orientale du Caire, Le Caire, 1974.

Nadan Amos, The Palestinian Peasant Economy under the Mandate, a Story of Colonial Bungling, Harvard University Press, Cambridge, 2006.

Nicault Catherine, "L'"orange de Jaffa" avant la Deuxième Guerre mondiale. Un fruit "palestinien" chargé de sens ", Archives Juives, vol. 47, n 1, 2014, p. 79-102.

Penslar Derek J., Zionism and technocracy: the engineering of Jewish settlement in Palestine, 1870-1918, Indiana University Press, Bloomington, 1991.

Picaudou Nadine, "Peuple juif/populations autochtones : les fondements de la domination britannique en Palestine ", dans Luizard Pierre-Jean, Le choc colonial et l'islam, La Découverte, Paris, 2006, p. 159-176. 
QuataERT Donald, Manufacturing and technology transfer in the Ottoman Empire, 1800-1914, Isis Press, Istanbul, 1992.

Soler Renaud, Edward Robinson (1794-1863) et l'émergence de l'archéologie biblique, Éditions Geuthner, Paris, 2014.

TEISCH Jessica, Engineering nature: water, development and the global spread of American environmental expertise, University of North Carolina Press, Chapel Hill, 2011.

Viollet Pierre-Louis, Histoire de l'énergie hydraulique. Moulins, pompes, roues et turbines de l'Antiquité au XX' siècle, Presses de l'école nationale des Ponts et chaussées, Paris, 2005.

\section{L'autrice}

Élisabeth Mortier, agrégée d'histoire, est doctorante en histoire contemporaine à Sorbonne-Université sous la direction du Professeur Catherine Mayeur-Jaouen. Sa thèse, à la croisée de l'histoire environnementale, de l'histoire des techniques et de l'histoire politique, porte sur les territoires de l'eau en Palestine rurale pendant la période de domination britannique (1917-1948). Derniers articles parus : " La figure héroïque du pionnier sioniste », Études rurales, n²03, 2019 et « Le "combat contre le désert et la sécheresse" : l'eau dans le Néguev et les projets sionistes à la fin du Mandat britannique en Palestine ", Revue d'histoire moderne et contemporaine, $n^{\circ}$ 66-4, 2019. 